\title{
Symplectic reduction and the problem of time in nonrelativistic mechanics
}

\author{
Karim Thebault*
}

November 23, 2010

\begin{abstract}
Symplectic reduction is a formal process through which degeneracy within the mathematical representations of physical systems displaying gauge symmetry can be controlled via the construction of a reduced phase space. Typically such reduced spaces provide us with a formalism for representing both instantaneous states and evolution uniquely and for this reason can be justifiably afforded the status of fundamental dynamical arena - the otiose structure having been eliminated from the original phase space. Essential to the application of symplectic reduction is the precept that the first class constraints (which feature in the Hamiltonian formalization of any gauge theory) are the relevant gauge generators. This prescription becomes highly problematic for reparameterization invariant theories within which the Hamiltonian itself is a constraint; not least because it would seem to render prima facie distinct stages of a history physically identical and observable functions changeless. Here we will consider this problem of time within non-relativistic mechanical theory with a view to both more fully understanding the temporal structure of these timeless theories and better appreciating the corresponding issues in relativistic mechanics. For the case of nonrelativistic reparameterization invariant theory application of symplectic reduction will be demonstrated to be both unnecessary; since the degeneracy involved is benign; and inappropriate; since it leads to a trivial theory. With this anti-reductive position established we will then examine two rival methodologies for consistently representing change and observable functions within the original phase space before evaluating the relevant philosophical implications. We will conclude with a preview of the case against symplectic reduction being applied to canonical general relativity (which will be examined more fully in future work).
\end{abstract}

${ }^{*}$ Centre for Time, University of Sydney. karim.thebault@gmail.com 


\section{Contents}

1 Introduction $\quad 2$

2 Mechanics with a fixed parameterization 4

2.1 Lagrangian mechanics . . . . . . . . . . . . . . . . . . . . 4

2.2 Hamiltonian mechanics . . . . . . . . . . . . . . . . . . . 5

2.3 Symplectic mechanics . . . . . . . . . . . . . . . . 5

2.4 Presymplectic geometry and symplectic reduction . . . . . . . . . 6

$\begin{array}{lll}3 & \text { Reductionism, haecceitism and gauge symmetry } & 9\end{array}$

4 Reparameterization invariant mechanics $\quad 11$

4.1 Extended Lagrangian mechanics . . . . . . . . . . . . . . . . . . . . 11

4.2 Extended Hamiltonian mechanics . . . . . . . . . . . . . . . . . . . 11

4.3 Jacobi's principle and timeless theory . . . . . . . . . . . . . . 12

4.4 Degeneracy, indeterminacy and triviality . . . . . . . . . . . . 14

5 Representing change and observables in timeless mechanics $\quad \mathbf{1 5}$

5.1 The emergent time strategy . . . . . . . . . . . . . . 16

5.2 The correlation strategy . . . . . . . . . . . . . . . . . 18

6 Interpretational Implications $\quad \mathbf{2 0}$

6.1 The relationalist vs substantivalist dispute with regard to time . . . . . 20

6.2 An ontology of timeless change? . . . . . . . . . . . . . . . . 23

$\begin{array}{lll}7 & \text { Preview of the case of general relativity } & 25\end{array}$

$\begin{array}{ll}\text { A Elements from differential geometry } & \mathbf{2 6}\end{array}$

$\begin{array}{lr}\text { B Acknowledgments } & \mathbf{2 7}\end{array}$

\section{Introduction}

Certain physical systems are such that the mathematical representation corresponding to them is degenerate - they contain what Redhead [2003] labels 'surplus structure'. By this we mean that the relevant equations of motion (together with the same initial data) produce multiple physically indistinguishable but mathematically distinct solutions. We can represent these formal redundancies in terms of groups of symmetry transformations on some space of possible configurations of the system and if these groups are suitably local we call them gauge symmetries. In order to determine a unique mathematical representation for a system displaying gauge symmetries it is the theory of constrained Hamiltonian systems (Dirac [1958a],[1964] or Henneaux and Teitelboim [1992]) was developed. This theory allows us to characterise the degeneracy precisely in terms of (first class) constraint functions on phase space and regulate it by providing equations that pick out dynamics that is independent of the action of the constraints. Geometrically Dirac's procedure for eliminating degeneracy within gauge theories can be understood in terms of a process of reduction from a constraint manifold within phase space with a presymplectic geometry to a reduced phase space with a symplectic geometry (Gotay and Nester [1978]) and philosophically this reduction process can 
be endowed with the significant role of providing a passage from a dynamical arena with excess representational structure to one which provides a direct representation of the true dynamical degrees of freedom (on this point see Belot [2007] and Butterfield [2007]).

The general theory of relativity explicitly features local redundancy in the form of diffeomorophisms invariance and can be cast into a constrained Hamiltonian formalism known as canonical general relativity (Dirac [1958b], Bergman [1961], Arnowitt et al. [1962], Misner et al. [1970]). Perplexingly, however, once rendered into constrained Hamiltonian form the degeneracy of the theory seems to become entangled with the dynamics since the canonical Hamiltonian is itself a first class constraint. Thus according Dirac's work it even though it effects the the transformations between three dimensional hypersurfaces that play the role of time in the theory the Hamiltonian should be interpreted as a gauge generator ${ }^{1}$. Furthermore, if we accept that the Hamiltonian is gauge generating then it would seem that we must classify as observable only functions which weakly commute with it (i.e. which have vanishing Poisson bracket with the constraint on the phase space surface it defines). This class of observables cannot vary along entire histories of a system and are therefore completely unable to change with respect to time. Correspondingly, from a geometric perspective application of symplectic reduction techniques to canonical general relativity is understood as leading to a reduced phase space which, despite having a symplectic structure, can no longer be understood as representing temporal evolution of either states or observables (see Belot and Earman [2001], Rickles [2008], Belot [2007]). This is the essence of the the problem of time in classical gravity - it is intimately connected to various issues that beset attempts to formulate a quantum theory of gravity and are grouped together as the problem of time in quantum gravity (Kuchař [1988], Isham [1992] and also Anderson [2010]).

The chain of argument leading to the classical problem of time is controversial. In particular, Kuchař ([1992]) and Barbour ([1994]) have argued that there are characteristics peculiar to the Hamiltonian constraint which mean we should not follow the standard procedure and treat it as gauge generating. On a similar note, Pons and Salisbury ([2005]) argue that Dirac's analysis is incomplete (Pons [2005]) since gauge symmetry groups should be more properly thought of as acting on the space of entire solutions rather than, as Dirac assumes, at a given time. Thus, under their analysis it is simply erroneous to identify the Hamiltonian (which acts on initial data points in order to create solutions) as a gauge generator. Also in this anti-Dirac spirit, Barbour and Foster ([2009]) have explicitly considered the case of Jacobi's theory which provides a useful model for general relativity since its reparameterization invariant action and vanishing Hamiltonian make it timeless in a fundamental sense. Contrary to Dirac's work they conclude that the Hamiltonian can be taken to generate genuine physical change and that observables that do not weakly commute with the Hamiltonian can be defined consistently.

The primary purpose of this paper is to examine these significant claims from both a technical and interpretive perspective within the context of the geometric presentation of the problem of time in non-relativistic mechanics. After providing a concise introduction to the relevant ideas from geometrical mechanics (additional material concerning differential geometry can be found in an appendix) we will presenting Dirac's argument for the classification of first class constraints as gauge generating in terms of a simplified

\footnotetext{
${ }^{1}$ The philosophical implications of accepting such a classification of the Hamiltonian are explicitly considered by Earman [2001]
} 
version of the symplectic reduction procedure. The supposed interpretational benefits for following such a reduction procedure will then be considered before we attempt explicitly to apply symplectic reduction to a class of nonrelativistic, reparameterization invariant theories (such as Jacobi's theory) within which the Hamiltonian is the only constraint. It will be argued - in support of Barbour and Foster - that the application of this geometric version of Dirac's work is inappropriate and this leaves open the question of how we should define both change and observables within non-relativistic reparameterization invariant mechanics. Utilizing the symplectic formalism that has been introduced we will then evaluate two rival positions that offer new methodologies for defining both change and observables - these will be designated emergent time strategy and the correlation strategy respectively ${ }^{2}$. We will then offer some thoughts as to the interpretative implications of both our negative result regarding symplectic reduction and of our two non-reductive schemes. Finally, we will conclude by providing a preview of the case of general relativity for which our non-relativistic work is expected to provide a useful framework.

\section{Mechanics with a fixed parameterization}

\subsection{Lagrangian mechanics}

We start with the specification of the set of $n$ independent variables, $q_{i} i=1 \ldots n$, which serve to characterise the properties of a mechanical system. Individually each of these variables can be taken to range over the set of real numbers, $\mathbb{R}$, and together they form an $n$ dimensional configuration space, $\mathcal{C}_{0}{ }^{3}$. At a given point $q \in \mathcal{C}_{0}$ we can define a tangent space $T_{q} \mathcal{C}_{0}$. The union of all the tangent spaces defined in $\mathcal{C}_{0}$ is called the tangent bundle $T \mathcal{C}_{0}$. The elements of the tangent bundle are pairs of configuration variables and vectors tangent to those variables. For formulations of mechanics with a fixed parameterizaton the parameter with which the tangent vectors are defined is unique and may be interpreted as time $t$ (this will prove not to be the case for the theories of mechanics considered in section 4). Thus we have $(q, \dot{q}) \in T \mathcal{C}_{0}$ with $\dot{q}=\frac{\partial q}{\partial t}$.

A curve within the tangent bundle, $\gamma_{0}: \mathbb{R} \rightarrow T \mathcal{C}_{0}$, will correspond to a history of a system - a sequence of configurations and velocities. The parameterization of the curve will be fixed up to a choice of origin by the distinguished time parameter $t$. This parameter can be taken to vary monotonically along each curve in configuration space. Clearly, for this picture to match up with the physics of the real world we need some restriction on which histories are nomologically possible. This is achieved by defining the Lagrangian, $L_{0}: T \mathcal{C}_{0} \rightarrow \mathbb{R}$, and the action, $I\left[\gamma_{0}\right]=\int_{\gamma_{0}} L_{0}\left[q_{i}, \dot{q}_{i}\right] d t=\int_{\gamma_{0}}(T-V) d t$, where $T$ and $V$ are kinetic energy and potential energy respectively. The extremisation of the action, $\delta I\left[\gamma_{0}\right]=0$, according to the principle of least action leads to the EulerLagrange equations, $\frac{\partial}{\partial t}\left(\frac{\partial L_{0}}{\partial \dot{q}_{i}}\right)=\frac{\partial L_{0}}{\partial q_{i}}$, that specify a set of paramterized solutions, $\left\{\gamma_{P S}\right\} \subset\left\{\gamma_{0}\right\}$, which uniquely determine the physically possible histories of the system given an initial point in $T \mathcal{C}_{0}$.

\footnotetext{
${ }^{2}$ The first has its originates with Kuchař [1992], Barbour [1994] and Barbour and Foster [2009] and the second with Rovelli ([1990], [1991], [2002], [2004]), Dittrich ([2006], [2008]) and Thiemann ([2007])

${ }^{3}$ The subscript 0 is used to distinguish the objects introduced here from those of the extended description of mechanics given insection 4 .
} 


\subsection{Hamiltonian mechanics}

An alternative formulation of mechanics in terms of first order equations is enabled by moving to the cotangent bundle of our configuration manifold, the phase space $\Gamma_{0}=T^{*} \mathcal{C}_{0}$. This is the union of all the cotangent spaces $T_{q}^{*} \mathcal{C}_{0}$. A point in phase space, $(q, p)$, consists of a point in our original configuration space, $q \in \mathcal{C}_{0}$, paired with a covector at $q, p \in T_{q}^{*} \mathcal{C}_{0}$. These covectors, which we call the conjugate momenta, are given by the Legendre transformation, $\mathcal{F} \mathcal{L}: T \mathcal{C}_{0} \rightarrow T^{*} \mathcal{C}_{0}$, which is the map between the configuration-velocity space and the phase space. It can be explicitly constructed using the definition of the canonical momenta, $p_{i}=\frac{\partial L}{\partial \dot{q}_{i}}$. To fix the dynamics we introduce the Hamiltonian functional, $H_{0}\left[q_{i}, p_{i}\right]=p^{i} q_{i}-L=T+V$, and derive Hamilton's equations, $\dot{p}_{i}=-\frac{\partial H_{0}}{\partial q_{i}}$ and $\dot{q}_{i}=\frac{\partial H_{0}}{\partial p_{i}}$. The relevant parameterized solutions $\bar{\gamma}_{P S}$ describe the system's dynamics unqiuely in the phase space $\Gamma_{0}$ and are isomorphic to the solutions $\gamma_{P S}$ in the configuration-velocity space $T \mathcal{C}_{0}$.

\section{$2.3 \quad$ Symplectic mechanics}

An elegant and more powerful characterisation of mechanical systems is provided by the symplectic approach (see Abraham and Marsden [1978], Arnold [1988] or Souriau [1997]). Symplectic is a Greek word first introduced in this context by Herman Weyl ([1939]). It means roughly 'plaited together' or 'woven' and a symplectic approach to mechanics involves the generalised description of the phase space used above in terms of a natural geometric language with the canonical momenta and configuration variables represented explicitly as intertwined.

Above we defined a covector as the dual of a tangent vector, similarly we can define a cotangent vector field or one-form as the dual of a tangent vector field. We can generalise these objects to define a $k$-form as a smooth section of the $k$ th exterior power of the cotangent bundle, $\Omega^{k}\left(T^{*} M\right)$. Of particular interest are two-forms which are functions $\Omega(x): T_{x} M \times T_{x} M \rightarrow \mathbb{R}$ that assign to each point $x \in M$ a skew-symmetric bilinear form on the tangent space $T_{x} M$ to $M$ at $x$ (Marsden and Ratiu 1994). We can transform a $k$-form into a $k+1$-form by the action of the exterior derivative, $\mathbf{d}: \Omega^{k}\left(\Gamma_{0}\right) \rightarrow \Omega^{k+1}\left(\Gamma_{0}\right)$. It is such that $\mathbf{d} f=d f, \mathbf{d}(\mathbf{d} \alpha)=0$ and $\mathbf{d}(f \alpha)=d f \wedge \alpha+f \mathbf{d} \alpha$ where $\alpha$ is a $k$-form and $d f$ is the differential of $f$.

Given a general cotangent bundle, $T^{*} M$, we can always define a corresponding Poincaré one-form ${ }^{4}, \theta$, in terms of a sum of products between a covector and the total differential of the vector it is paired with. Thus for our phase space, $\Gamma_{0}$, the Poincaré one form is $\theta=p_{i} d q^{i}$. If we then take the exterior derivative we get a two form:

$$
\omega_{0}=\mathbf{d} \theta=\mathbf{d}\left(p_{i} d q^{i}\right)=d p_{i} \wedge d q^{i}
$$

This two form is called a symplectic two form and is both closed $\left(\mathbf{d} \omega_{0}=0\right)$ and non-degenerate (if $\omega_{0}\left(X_{f}, X_{g}\right)=0$ for all $X_{f} \in T M$ then $\left.X_{g}=0\right)$ ). A manifold endowed with a symplectic two form constitutes a symplectic geometry $\left(M, \omega_{0}\right)$. Significantly, if we are given a smooth function, $f$, on a manifold endowed with a symplectic two form then we immediately define uniquely a smooth tangent vector field $X_{f}$ through the map $f \longmapsto X_{f}$ given to us by $\omega_{0}\left(X_{f}, \cdot\right)=\mathbf{d} f$. The uniqueness of the vector field is guaranteed by the non-degeneracy of $\omega_{0}$.

\footnotetext{
${ }^{4}$ See von Westenholz [1978], pp.392-394 for more details.
} 
The relation between symplectic geometry and the Hamiltonian theory of mechanics outlined above can be seen immediately since Hamilton's equations can be written:

$$
\left(\dot{q}_{1}, \ldots, \dot{q}_{n}, \dot{p}_{1}, \ldots \dot{p}_{n}\right)\left(\begin{array}{cc}
0 & I \\
-I & 0
\end{array}\right)=\left(\frac{\partial H_{0}}{\partial q_{1}}, \ldots, \frac{\partial H_{0}}{\partial q_{n}}, \frac{\partial H_{0}}{\partial p_{1}}, \ldots, \frac{\partial H_{0}}{\partial p_{n}}\right)
$$

where $I$ is the $n \times n$ identity matrix. This expression is an unknown vector multiplied by a matrix and set equal to known vector. It is equivalent to

$$
\omega_{0}\left(X_{H_{0}}, \cdot\right)=\mathbf{d} H_{0}
$$

which is an unknown tangent vector field (the Hamiltonian vector field $X_{H}$ ) contracted with a two form and set equal to the exterior derivative of a the Hamiltonian, $H$. Thus we can see Hamilton's equations have an immediate connection with symplectic geometry. The dynamics of a system can be totally specified by the triple $\left(\Gamma_{0}, \omega_{0}, H_{0}\right)$, where $\Gamma_{0}$ is our phase space manifold (cotangent bundle), $\omega_{0}$ is the symplectic two form, and $H_{0}$ is the Hamiltonian function on $\Gamma_{0}$. Together these three elements fix the value of the Hamiltonian vector field, $X_{H_{0}}$. It is the integral curves of this vector field that correspond to the parameterized phase space solutions $\bar{\gamma}_{P S}$ that we associated with the physical histories above.

The Hamiltonian vector field that we have just defined is unique. This implies that it will generate a unique $\mathbb{R}$-action on phase space. This $\mathbb{R}$-action and the associated flow, are what we conventionally identify as temporal evolution since they take us from a point in phase space (instantaneous state of a physical system) to a second point (state) that is $t$ units along a solution (physical history). Thus, we see that there is a intimate connection between the Hamiltonian and time.

This connection is made even more explicit by the introduction of the Poisson bracket which is a special case of the Lie bracket defined that can be defined via the symplectic two form for any pair of functions, $f, g \in C^{\infty}\left(\Gamma_{0}\right)$, as:

$$
\{f, g\}:=\omega_{0}\left(X_{f}, X_{g}\right)=\sum_{i=1 . . n} \frac{\partial f}{\partial q_{i}} \frac{\partial g}{\partial p_{i}}-\frac{\partial f}{\partial p_{i}} \frac{\partial g}{\partial q_{i}}
$$

The Poisson bracket can be related to the action of a vector field on a smooth function:

$$
\{f, g\}=X_{g}(f) \equiv d f\left(X_{g}\right) \equiv \mathcal{L}_{X_{g}}(f)
$$

This means that if we take the Poisson bracket of the Hamiltonian with an arbitrary smooth function we will get the change of this function along the flow defined by the Hamiltonian vector field. This is equal to the variation of the function with respect to the flow parameter of $X_{H_{0}}$ which is, of course, how change with respect to time is represented:

$$
\left\{f, H_{0}\right\}=X_{H_{0}}(f)=\frac{d f}{d t}=\dot{f}
$$

Conversely, the commutation condition $\left\{f, H_{0}\right\}=0$ indicates that a function is conserved - it does not change with respect to time.

\subsection{Presymplectic geometry and symplectic reduction}

A physical system within which a Lie group, $G$, acts on the tangent bundle, $T \mathcal{C}_{0}$, such that the Lagragian, $L$, is invariant and the group is local (i.e. it can be parameterized 
in a natural way by a family of arbitrary functions on space-time) is said to display a gauge symmetry. In such systems the assumption that the Legendre transformation is an isomorphism ${ }^{5}$ which was implicit in our construction of mechanics above no longer holds. This is because the bijectivity of the map $\mathcal{F} \mathcal{L}: T \mathcal{C}_{0} \rightarrow T^{*} \mathcal{C}_{0}$ is dependent on the Lagrangian being such that it determines tangent vectors $\dot{q}$ uniquely through the definition of the canonical momenta. Gauge symmetries manifestly subvert this since we have that $L\left(q^{\prime}, \dot{q}^{\prime}\right)=L(g q, g \dot{q})=L(q, \dot{q})$ for $\forall g \in G$. In phase space terms the existence of a gauge symmetry group corresponds to the $p_{i}$ 's and $q_{i}$ 's not all being independent - there exists some functional relationship between them of the form $\varphi(p, q)=0$. We call such functions constraints ${ }^{6}$.

Geometrically we can understand the collection of all the constraints, $\varphi_{j} j=1, \ldots m$, as defining an $m$ dimensional sub-manifold, $\Sigma=\left\{(p, q) \in \Gamma_{0} \mid \forall_{j}: \varphi_{j}(p, q)=0\right\}$, within phase space, $\Gamma_{0}$, that we call the constraint surface. The phase space itself will, as in the unconstrained case, have a symplectic geometry characterised by the pair $\left(\Gamma_{0}, \omega\right)$ where $\omega$ is again a closed and non-degenerate two form constructed by taking the total differential of the Poincaré one form $\theta=p_{i} d q^{i}$. However, points in this space which do not lie on the constraint surface will not correspond to physically possible states since they constitute solutions which violate the gauge symmetry (they are inaccessible or merely unphysical in the language of Rickles [2004]). It is the geometry particular to the class of points lying on the constraint surface that is nomologically significant.

We can characterise the geometry of the constraint surface explicitly by first restricting $\theta$ to $\Sigma$ to get a new characteristic one form, $\tilde{\theta}=\theta_{\left.\right|_{\Sigma}}$. The total derivative of $\tilde{\theta}$ will then give us a two form $\tilde{\omega}=\mathbf{d} \tilde{\theta}$ which endows the constraint manifold with the geometry $(\Sigma, \tilde{\omega})$. This new two form will be closed but whether it is degenerate or not depends on the particular properties of the constraint surface itself. In cases where it is non-degenerate we again have a symplectic geometry and the dynamics is as described above only now with the triple $\left(\Sigma, \tilde{\omega}, \tilde{H}_{0}\right)$ defining the system (where $\tilde{H}_{0}: \Sigma \rightarrow \mathbb{R}$ is the is the restriction of $H_{0}$ to $\Sigma$ ).

In the case that $\tilde{\omega}$ is degenerate, however, we have a presymplectic geometry and our regular description of dynamics is no longer available to us. This is because presymplectic geometries have a degenerate structure that does not allow us to associate a unique vector field with every smooth function and so $\tilde{\omega}\left(X_{\tilde{H}_{0}}, \cdot\right)=\mathbf{d} \tilde{H}_{0}$ will only give us an equivalence class of Hamiltonian vector fields. This means that we are not provided with a straightforward characterisation of time evolution either via a unique $\mathbb{R}$-action or by the usual Poisson bracket with the Hamiltonian. Even more worryingly, the existence of local symmetry groups allows for indeterministic (or more properly underdetermined) evolution since at a given point the degeneracy of the Hamiltonian vector field allows for multiple mathematically distinct but dynamically equivalent solutions irrespective of the path leading up to that point. Thus, it would seem that the degeneracy inherent in presymplectic geometries is of a pernicious variety such that we can no longer establish a direct representational relationship between the relevant mathematical and ontological objects - there is no longer a one-to-one correspondence between the phase space solutions and the physical histories which are distinguished by unique values of the action and so our theory is underdetermined.

To get a better hold on the nature of this degeneracy we can define the null tangent vector space $N_{x} \subset T_{x} \Sigma$ as the collection of vectors that satisfy the equation $\tilde{\omega}(X, \cdot)=0$. This is equivalent to the null space or kernel, $\operatorname{Ker}(\tilde{\omega})$, of the presymplectic form.

\footnotetext{
${ }^{5} \mathrm{~A}$ map that is both bijective (injective and surjective) and a homomorphism (structure preserving).

${ }^{6}$ These particular constraints are known as primary constraints. See footnote 20 for details.
} 
A kernel of dimension greater than zero is characteristic of the non-trivial structure presymplectic form just as a kernel of dimension equal to zero is characteristic of the trivial structure of the symplectic form. An equivalence relation between two points $x, y \in \Sigma$ can be defined based upon the condition of being joined by a curve, $\bar{\gamma}: \mathbb{R} \rightarrow \Sigma$, with null tangent vectors. Sets of points for which this equivalence relation holds are submanifolds called gauge orbits, $[x]$, and we say that the action of our presymplectic form is to partition phase space into these orbits. Equivalently we can say that the orbits are defined by the integral curves of the null vector field of $\tilde{\omega}$. The non-uniqueness that we understood in terms of the existence of gauge orbits is, therefore, also characterised by $\operatorname{Ker}(\tilde{\omega})$.

Critically for our purposes the quotient $\Pi_{R}=\Sigma / \operatorname{Ker}(\tilde{\omega})$ will necessarily be both symplectic and a manifold. The first is assured since the quotient we are taking is of a manifold by a sectional foliation ${ }^{7}$. The second is assured because the quotient is of a presymplectic manifold with respect to the kernel of its own presymplectic form and it can be shown that this implies that the resulting quotient manifold will be endowed with a closed two form with a kernel of zero dimension - i.e. it will have a symplectic geometry $^{8}$. We can now represent evolution in terms of a unique $\mathbb{R}$-action defined in $\Pi_{R}$. We call $\Pi_{R}$ the reduced phase space and using the projection map $\pi: \Sigma \rightarrow \Pi_{R}$ can define the symplectic geometry $\left(\Pi_{R}, \omega_{R}, H_{R}\right)$ where $\omega_{R}$ is the two form whose pullback to $\Sigma$ by $\pi$ is $\tilde{\omega}$ (i.e. $\tilde{\omega}=\pi^{*} \omega_{R}$ where $\pi^{*}: \Pi_{R} \rightarrow \Sigma$ ). An equation of the form $\omega_{R}\left(X_{H_{R}}, \cdot\right)=\mathbf{d} H_{R}$ then gives us a unique Hamiltonian vector field along with the associated Poisson bracket and $\mathbb{R}$-action that allows us to uniquely represent both time and the physical histories uniquely within our formalism.

The pullback by $\pi$ also allows us to consider the properties that smooth functions on the reduced phase space will have with respect to the constraint manifold. Given such a function, $f_{R} \in C^{\infty}\left(\Pi_{R}\right)$, we can define $f_{\Sigma} \in C^{\infty}(\Sigma)$, by $f_{\Sigma}=\pi^{*} f_{R}$. Since points connected by a gauge orbit on $\Sigma$ will be represented by a single point on $\Pi_{R}$ we have that $f_{\Sigma}$ will be constant along such gauge orbits. We can also talk about functions on the full phase space as being constant along gauge orbits. Since the constraints are by definition functions of the form $\varphi_{j}: \Gamma_{0} \rightarrow 0$ the symplectic form on phase space will associate them each with a vector field $X_{\varphi_{j}}$. If we then take the Poisson bracket between them and an arbitrary function, $f \in C^{\infty}\left(\Gamma_{0}\right)$, we will have $\left\{f, \varphi_{j}\right\}=\omega\left(X_{f}, X_{\varphi_{j}}\right)$. On the constraint surface it must be the case that the $X_{\varphi_{j}}$ coincide with the null vector fields $N$ - the integral curves of which are the gauge orbits. So, given that on the constraint surface $f$ must be a function which is unchanging along the gauge orbits, the definition of the Poisson bracket implies that the expression $\left\{f, \varphi_{j}\right\}$ must vanish on the constraint surface - i.e. we have that $\left\{f, \varphi_{j}\right\} \approx 0$, where the weak equality is understood to mean zero upon the constraint surface.

We can therefore distinguish a class of functions on phase space, Dirac-Bergman observables, by the satisfaction of three equivalent conditions:

1. Constancy along gauge orbits on the constraint manifold

2. Weakly commuting with all the constraints

3. Equivalence to a function on the reduced phase space

\footnotetext{
${ }^{7}$ See Souraiu [1997] p.42 and pp. $82-3$. It is a sectional foliation because the orbits which partition $\Sigma$ constitute manifolds which are suitably transverse.

${ }^{8}$ See Souraiu [1997] theorem 9.10
} 
The name observable seems sensible since it is only these functions that are specified uniquely for every value of the flow parameter defined by the vector field generated by the reduced Hamiltonian, $H_{R}$. Thus, given our reliance on an underlying symplectic structure to define time, precise restrictions are placed upon the mathematical objects with which we would want to associate physical quantities.

This idea of passing from a presymplectic to a symplectic manifold by quotienting with respect to the kernel of the presymplectic form is what we will call symplectic reduction and has an important connection ${ }^{9}$ with Dirac's theory of constraints. In particular, in cases (such as those considered in the next section) where there is only one primary constraint and no secondary constraints $^{10}$ the application of symplectic reduction is identical to following the Dirac procedure in that it leads to the same conditions on observable functions we have just outlined. A theory in which all first class primary constraints are gauge generating is said to obey Dirac's theorem (Barbour and Foster [2009]) and we can therefore say that the applicability of symplectic reduction is equivalent to satisfaction of Dirac's theorem in all theories with a single primary constraint.

\section{Reductionism, haecceitism and gauge symmetry}

The identification between gauge theories treated according to Dirac's constraint procedure and the re-construction of such theories in terms of reduced phase spaces arrived at via symplectic reduction has important interpretational consequences. As we have seen above conventional Hamiltonian mechanics can be characterized in terms of a phase space which has a symplectic geometry and within which solutions (the integral curves of the Hamiltonian vector field) are in one-to-one correspondence with physical histories. In these circumstances it seems natural to identify the phase space as a possibility space since each point can be considered to represent a distinct possible instantaneous physical state and each curve a distinct possible physical history. On the other hand, when we have a constrained Hamiltonian system the relevant phase space is clearly not a suitable candidate for a possibility space it contains inaccessible points (i.e. those not on the constraint surface) which can not be thought of as representing physically possible states. Furthermore, even if we exclude such points and focus on the accessible section of phase space (i.e. consider only points on the constraint surface) then we again do not have a natural candidate for a possibility space since the weaker presymplectic geometry only equips us with an equivalence class of solutions corresponding to each physical history. This leaves the theory open to pernicious underdetermination such that if points are identified as representing distinct instantaneous states then specifying an initial sequences of states fails to uniquely distinguish future states. Since the class of classical constrained Hamiltonian theories features theories, such as electromagnetism, which are manifestly deterministic in the sense of giving unique predictions for all measurable quantities the appearance of indeterminism should be seen to be interpreted as a sign of inadequacy in our representative formalism ${ }^{11}$ - we cannot identify

\footnotetext{
${ }^{9}$ See Gotay et al. [1978] and Pons et al. [1999] for explicit examination of this connection

${ }^{10}$ Primary constraints are those that arise directly from the fact that the conjugate momenta are not independent functions of the velocities. Secondary constraints arise from the application of consistency conditions that ensure the primary constraints are preserved.

${ }^{11}$ As pointed out by Belot and Earman ([2001]) the only other alternative in such circumstances would be to accept that there exist physically real quantities that are not measurable - although potentially consistent, this would seem like a very unnatural approach and would require us to construct a highly
} 
the constraint surface as a possibility space in a conventional sense .

Rather, we could treat it as an unconventional possibility space by weakening the representative connection between points on the constraint surface and instantaneous states. The classic philosophical strategy to enable such a weakening would be to adopt some form of anti-haecceitism. If we define a haecceitist as someone who believes that two objects can remain distinct without having any qualitative differences then the standard literal way of interpreting a possibility space - i.e. each point represents a distinct instantaneous state - can be be understood as haecceitist since it allows for two points to represent states which are physically indistinguishable and yet still distinct. When applied to the constraint manifold of a gauge theory such an approach becomes difficult because its combination with the presentence of pernicious undertermination forces us into interpreting empirically deterministic theories as ontologically indeterministic. An anti-haecceitist, on the other hand, denies primitive identity and so can disavow the idea that two gauge related points in a possibility space represent distinct states. Thus by adopting anti-haecceitism we can relieve ourselves of the burden of having to endorse ontological indeterminism by instituting a many-to-one relationship between points on the constraint surface and instantaneous states.

Although providing space for a viable interpretation of the possibility space structure found in gauge theory the anti-haecceitist approach does nothing about removing what would seem like otiose mathematical structure - to dispense with this surplus structure we need to move the reduced phase space. Now, this space has obvious interpretational benefits since, as seen above, if all goes well the reduced space will be a symplectic manifold with the integral curves of the reduced Hamiltonian vector field naturally identified as representing physical histories and points as representing physically distinct instantaneous states. Thus the reduced space will, by definition, not feature any undertermination and if we endow it with the privileged status as our fundamental possibility arena we reap the reward of recovering the ability to use our conventional representational structure for theories which display gauge symmetry. Since we have regained a one-to-one correspondence between possibility space points and physically distinct instantaneous states the haeccitism/anti-haecceitism distinction becomes moot and our representative framework is happily freed from metaphysical commitments concerning the nature of identity and modality. The superiority of, when possible, reduction as an interpretational stance has been advocated principally by Gordon Belot and John Earman (Belot [1996], [2000], [2003], [2003]; Earman [2001]; and Belot and Earman [1999],[2001]). We will call it the reductionism with regard to constrained Hamiltonian theory and a close association can be made between it and Dirac's theorem as defined above - in fact it would seem fair to say that the reductive philosophical stance is the natural interpretational consequence of a strict reading of Dirac's theorem. Arguments towards the non-applicability of symplectic reduction (corresponding to Dirac's theorem) for the specific case of non-relativistic reparameterization invariant mechanics will be the major preoccupation of the next section. Section 5 will then focus on techniques for representing time and change within the unreduced phase space before we will return, in section 6 , to interpretational issues connected with both reductionism/anti-reductionism and haeccitism/anti-haecceitism.

unorthodox account of the concept of measurement 


\section{Reparameterization invariant mechanics}

\subsection{Extended Lagrangian mechanics}

The description of mechanics and gauge symmetry given thus far has made use of a distinguished background parameter; time $t$. Within the Lagrangian scheme this parameter was associated with both the tangent vectors or velocities, $\dot{q}=\frac{\partial q}{\partial t} \in T \mathcal{C}_{0}$, and with the preferred parameterization of the solutions, $\gamma_{P S}: \mathbb{R} \rightarrow T \mathcal{C}_{0}$. An alternative methodology for constructing mechanical theory is to instead treat time as an additional coordinate, $q_{0}=t$, in a $n+1$ dimensional extended configuration space, $\mathcal{C}=\mathbb{R} \times$ $\mathcal{C}_{0}$. Velocities in this space are then defined for all of the $q_{\mu} \in \mathcal{C}$ by differentiation with respect to an arbitrary parameter $\tau$ so we have that $q_{\mu}^{\prime}=\frac{d q_{\mu}}{d \tau},\left(q_{\mu}, q_{\mu}^{\prime}\right) \in T \mathcal{C}$. This arbitrary parameter is also taken to vary monotonically along curves in extend configuration space, $\gamma: \mathbb{R} \rightarrow T \mathcal{C}$. Following Lanzcos $(1966, \S 5)^{12}$ we can use an extended Lagrangian, $L_{e x}\left[q_{\mu}, q_{\mu}^{\prime}\right]: T \mathcal{C} \rightarrow \mathbb{R}$ to define an action of the form:

$$
I=\int_{\gamma} d \tau L_{e x}\left[q_{\mu}, q_{\mu}^{\prime}\right]=\int_{\gamma} d \tau\left(\frac{\bar{T}}{q_{0}^{\prime}}-q_{0}^{\prime} V\right)
$$

where $\bar{T}=q_{0}^{\prime 2} T$ and all masses are set to unity.

An important property of the extended Lagrangian is that it is homogenous of degree one in the extended set of velocities $q_{\mu}^{\prime}$ : for some positive number $k$ the transformation $q_{\mu}^{\prime} \rightarrow k q_{\mu}^{\prime}$ implies $L_{e x}\left[q_{\mu}, q_{\mu}^{\prime}\right] \rightarrow k L_{e x}\left[q_{\mu}, q_{\mu}^{\prime}\right]$. This means that the action of our theory will be invariant under re-scalings of the parameter $\tau$. Theories which display such a dynamic insensitivity to parameterization are said to be reparameterizations invariant. The interpretation of this theory will be non-standard since reparameterization is a symmetry of the action which maps between distinct solutions in the extended configuration space - this is because the velocities are parameterization dependent. Thus these solutions cannot be used to straightforwardly characterise physical histories as in $\S 3.1$.

\subsection{Extended Hamiltonian mechanics}

In correspondence with $\S 3.2$ we can define an extended phase space as the cotangent bundle to our extended configuration manifold, $\left(q_{\mu}, p_{\mu}\right) \in \Gamma=T^{*} \mathcal{C}=T^{*}\left(\mathbb{R} \times \mathcal{C}_{0}\right)$, with $p_{\mu}=\frac{\partial L_{e x}}{\partial q_{\mu}^{\prime}}$. The relevant Hamiltonian functional, $H_{e x}\left[q_{\mu}, p_{\mu}\right]: \Gamma \rightarrow \mathbb{R}$ takes the form:

$$
H_{e x}\left[q_{\mu}, p_{\mu}\right]=p^{\mu} q_{\mu}^{\prime}-L_{e x}\left[q_{\mu}, q_{\mu}^{\prime}\right]
$$

which is homogenous of degree one in the set of extended velocities and defines a reparameterization invariant action

$$
I=\int_{\gamma} d \tau\left(p^{\mu} q_{\mu}^{\prime}-H_{e x}\left[q_{\mu}, p_{\mu}\right]\right)
$$

By definition we have that the momentum conjugate to time is:

$$
p_{0}=\frac{\partial L_{e x}}{\partial q_{0}^{\prime}}=L_{0}-\frac{\partial L_{0}}{\partial \dot{q}_{i}} \frac{q_{i}^{\prime}}{t^{\prime}}=-H_{0}
$$

\footnotetext{
${ }^{12}$ Also see Johns [2005], §11 and $\S 12$ and Rovelli [2005], §3.1)
} 
which means the extended Hamiltonian is equivalent to:

$$
\begin{aligned}
H_{e x}\left[q_{\mu}, p_{\mu}\right] & =t^{\prime}\left(p_{0}+H_{0}\right) \\
& =0
\end{aligned}
$$

The Hamiltonian is therefore a constraint and the dynamics of our theory will be defined upon a surface within extended phase space, $\Sigma=\left\{x \in \Gamma: H_{e x}(x)=0\right\}$. The geometry of the constraint surface is given (as above) by taking the restriction of the relevant Poincaré one form, $\theta=p_{\mu} d q^{\mu}$, to $\Sigma$ :

$$
\theta_{\mid \Sigma}=p_{i} d q^{i}-H_{0} d t
$$

and taking the total differential to get a two form $\tilde{\omega}=\mathbf{d}\left(\theta_{\mid \Sigma}\right)$ with highly non-trivial structure $^{13}$.

Significantly, this two form is closed and degenerate. Thus the dynamics of extended mechanics is framed within a presymplectic geometry, $(\Sigma, \tilde{\omega})$. That this should be the case can be seen quite simply since our definition of a degenerate two form is equivalent to Hamiltons equations of motion with a zero Hamiltonian:

$$
\begin{aligned}
\tilde{\omega}(X, \cdot) & =d H_{e x} \\
& =0
\end{aligned}
$$

The immediate consequence of the degeneracy is that no unique Hamiltonian vector field is defined within the constraint surface and thus that we cannot define an unique temporal $\mathbb{R}$-action or flow. Correspondingly, our equation of motion (14) is only solvable up to an arbitrary factor ${ }^{14}$ meaning that the dynamical solutions can only be unparameterized curves in the tangent bundle $\bar{\gamma}_{U P S}$.

The question is then; can we now simply follow a symplectic reduction procedure and then avail ourselves of the standard description of time, change and observable functions? Or does reparameterization have some unusual feature that necessitates a different approach? To tackle these issues we need to take a closer look at the physical interpretation of both time and its conjugate momentum and in doing so construct a more elegant and general version of reparameterization invariant mechanics.

\subsection{Jacobi's principle and timeless theory}

We can associate the time coordinate $t\left(q_{0}\right)$ in extended mechanics with the value taken by a clock external to our mechanical system. In the case of an open system such an interpretation would seem appropriate; but what about if the system is a closed subsystem of the universe? - or even the universe as a whole? In this case there is clearly no physical basis for an external clock and as such we would look to eliminate $t$ as an independent variable. We can do this by the process of Routhian reduction ${ }^{15}$ which serves to eliminate a cyclic independent variable (i.e. one which only appears in the Lagrangian as a velocity) by using the equations of motion to set its conjugate momentum equal to a constant. Since we have seen above that the conjugate

\footnotetext{
${ }^{13}$ This should come as no surprise as this two form must encode the full structure of the constraint and, since this constraint is the Hamiltonian, therefore the dynamics.

${ }^{14}$ This is because it can be though of as linear homogenous equation that only determines the velocities up to a scaling factor applied everywhere along a solution

${ }^{15} \mathrm{~A}$ fuller discussion of Routhian reduction in general, and in this case in particular, is given by Lanzcos ([1966] §5) and Arnold ([1988], §3.2).
} 
momentum to time is equal to minus the un-extended Hamiltonian of the system we will give the physical interpretation of the constant involved as minus the total energy, $E$, of the system. Setting the energy as equal to a constant is of course justified for a closed system. Explicitly, following Lanzcos $([1966], \S 5)$, the Jacobi action is given by

$$
I=\int_{\gamma_{0}} d \tau 2 \sqrt{(E-V) T}
$$

This action can be understood as defining geodesics in the un-extended space, $T \mathcal{C}_{0}$, without making any reference to time or parameterization - as such it is reparameterization invariant. We can define the lapse as:

$$
N=\sqrt{\frac{T}{(E-V)}}
$$

The Jacobi Hamiltonian (Barbour and Foster [2009], p.7), $H_{J}: T^{*} \mathcal{C}_{0} \rightarrow \mathbb{R}$ can then be expressed as:

$$
H_{J}=\sum_{i} p_{i} \cdot q_{i}^{\prime}-L_{J}=N h
$$

where

$$
h=\frac{1}{2} \sum_{i} p_{i} \cdot p_{i}+V-E=0
$$

This is again a first class primary constraint. In fact it is the same constraint as was encountered in extended mechanics merely with $p_{0}$ replaced by $-E$ and the multiplier $t^{\prime}$ replaced by $N$. Thus, reparameterization invariant theories of mechanics have a Hamiltonian of the form

$$
H=N h
$$

where $N$ is a arbitrary multiplier, the choice of which determines the parameterization, and $h$ is some function of the conjugate variables that is equal to zero. Such timeless theories will inevitably be constrained Hamiltonian theories with the Hamiltonian itself playing the role of the constraint. Thus the geometry of the constraint surface will be dictated by the two form $\omega=\mathbf{d} \theta=\mathbf{d}\left(\theta_{\left.\right|_{\Sigma}}\right)$ where $\Sigma=\{x \in \Gamma: H=0\}$.

This two form will in general be closed and it will also be degenerate since it has a null direction associated with the Hamiltonian constraint. The integral curves of this vector are the gauge orbits of $\omega$ on $\Sigma$. However, since this null vector field on the constraint surface is generated by the Hamiltonian we could also argue that $\omega(X)=0$ is the equation of motion ${ }^{16}$. Since the integral curves of the kernel of the presymplectic form can be shown to be unique solutions we have the strange situation in timeless mechanics such that the gauge orbits correspond to the physical histories! The question of how we are to interpret this unusual geometry where degeneracy and dynamics are so closely interwoven is far from trivial and shall occupy us for the remained of this paper. To go forward, however, we must go back and reconsider the connection between presymplectic geometry and local symmetry groups.

\footnotetext{
${ }^{16}$ This can be explicitly seen for the case of the simple pendulum system used by Rovelli [2004] to illustrate both extended mechanics ( $\S 3.1$ pp.104-105) and Jacobi's theory ( $\S 3.2$ pp.109-111 for Jacobi's theory - n.b. he refers to it non-standardly as relativistic mechanics).
} 


\subsection{Degeneracy, indeterminacy and triviality}

In our initial discussion of presymplectic geometry we associated the degeneracy encountered with a group of local or gauge symmetries arising on the tangent bundle to some configuration-velocity space, $T \mathcal{C}$. These symmetries were taken to be such that they allow for multiple points to be associated with the same value of the Lagrangian and thus ensured that the Legendre map, $\mathcal{F} \mathcal{L}: T \mathcal{C} \rightarrow T^{*} \mathcal{C}$, was not an isomorphism (a bijective homomorphism) since in such a situation it will generically neither be injective nor surjective. In the case of reparameterization invariant theory the relevant symmetry group is of course that of reparameterizations. It can be seen to be different to the generic gauge group considered in $\S 3.4$ in two important respects. First, since it relates points that differ in terms of parameterization it is strictly a symmetry of the action rather than the Lagrangian. Second, although it also leads to a Legendre transformation that is again not bijective (since it is not injective) the action of the reparameterization group is such that the conjugate momenta are not affected by rescaling the parameter. Thus, distinct points on the tangent bundle which can be mapped from one to another by the action of the reparameterization group will correspond to single points on the cotangent bundle. We therefore have that the structure of our phase space is such that paths through it are invariant under reparameterizations. The degeneracy present does not then lead to the type of pernicious underdetermination which was encountered in the construction of presymplectic mechanics considered in §3.4. Rather it takes us between vector fields that are equivalent up to scaling by a multiplicative factor corresponding to the parameterization. Our primary motivation for the application of the symplectic reduction procedure is therefore removed since there is no possibility of pernicious indeterminism.

We still, however, have the problem of representing change within the presymplectic constraint surface $(\Sigma, \omega)$ - one would like to be able to associate the Hamiltonian with a unique vector field and therefore be able to establish a unique flow with which we can associate evolution. The most obvious way to do this would be to find an underlying symplectic manifold within the timeless theory - thus it may be worth trying to symplectically reduce such theories even without a pressing theoretical need to. However, as pointed out above, timeless theories have a geometry such that what we would normally call the gauge orbits (since they are the sets of points connected by parameterization rescalings) are also the usual candidates for the solutions in phase space (since they are generated by the Hamiltonian). Thus, the reduction procedure whereby we quotient out the orbits of $\omega$, will leave us with a reduced phase space, $\Pi_{R}=\Sigma / \operatorname{Ker}(\omega)$, without any meaningful notion of evolution - it consists of unconnected points each of which can only gain meaning when referred back to the entire history on the constraint surface to which they correspond. Moreover, since the space is equipped only with a trivial Hamiltonian function there is no sense in which the reduced phase space symplectic form, $\omega_{R}$, found in reparameterization invariant theories of mechanics can play any meaningful role - even in generating maps between points in the reduced space. Thus, representationally $\Pi_{R}$ alone is only equipped to descirbe trivial universes consisting of one static configuration (Maudlin [2002] makes a similar point). Furthermore, since $\omega_{R}$ is defined only in virtue of the constraint surface via $\omega=\pi^{*} \omega_{R}$ there is a sense in which it could be said to have no more than a purely formal existence ${ }^{17}$.

It could be argued (see Belot [2007] p.78) in this context that points in the reduced phase space should be taken to describe entire dynamic solutions and therefore that

\footnotetext{
${ }^{17}$ Rovelli's (2005) treatment introduces $\omega_{R}$ as $\omega_{p h}$ (p.111) but fails to make any use of it.
} 
the space is not representationally trivial. In normal circumstances it is reasonable to interpret the reduced phase space, $\Pi_{R}$, resulting from the application of symplectic reduction as a space of instantaneous initial data states, $\mathcal{I}$. This follows from the fact that for any curve $\gamma_{P S}$ in the space of gauge invariant solutions to the Euler-Lagrange equations $\mathcal{S}_{R}$ we can define a set of isomorphisms between $\Pi_{R}$ and $\mathcal{S}_{R}$ such that for each value of the curves parameterization there will be a map uniquely picking out a point in $\Pi_{R}$ with corresponding value of the Hamiltonian flow parameter ${ }^{18}$. However, for the case of nonrelativistic ${ }^{19}$ timeless theory there is only a single canonical isomorphism defined between points in the reduced phase space and the unparameterized gauge invariant solutions, $\gamma_{U P S}$. Thus we can see why one might think the representational role of $\Pi_{R}$ should be modified such that it becomes identical to that of $\mathcal{S}_{R}$. But such a move has highly nontrivial consequences for how we must interpret the unreduced phase space and is therefore difficult to countenance. In particular, if $x_{R} \in \Pi_{R}$ is a solution then given a point on the constraint manifold in the unreduced phase space, $x \in \Sigma$, we must interpret the relevant 'gauge' orbit, $[x]: \Sigma \rightarrow \mathbb{R}$, as an equivalence class of solutions. This interpretation cannot hold since these orbits are equivalent to solutions themselves rather than equivalence classes of solutions. Thus, in nonrelativistic timeless theory at least the representational role of the unreduced phase space cannot be in describing entire histories - we cannot treat it as a primitive arena for representing our fundamental ontology. Rather, any status it can be given as a history space is purely parasitic on the pull-back map to the unreduced space and it is fallacious to argue that the isomorphism that exists between $\mathcal{S}_{R}$ and $\Pi_{R}$ must confer representational equivalence between these two very different mathematical structures.

It would seem therefore that we have established two examples of mechanical theory within which the presence of a first class constraint does not indicate that a symplectic reduction is appropriate. This means that Dirac's theorem (first class constraints generate gauge symmetry) does not hold for the timeless theories considered and is therefore not generally valid in its original form ${ }^{20}$.

\section{Representing change and observables in timeless me- chanics}

The essential point established by our argument thus far is that the unreduced phase space of a timeless system (i.e. one in which the Hamiltonian is a constraint) is such that we cannot interpret it using the convectional machinery of constrained Hamiltonian mechanics. Although, as in the generic case, points not on the constraint surface must be classified as inaccessible states; it has been demonstrated that, unlike in the generic case, the difference between points connected by the orbits generated by the constraint on the constraint surface itself cannot be classified as purely unphysical gauge without trivialising the theory. Thus the geometric structure of timeless theories leads us into acute problem of representing change since we cannot avail ourselves of the conventional

\footnotetext{
${ }^{18}$ The geometric structure of such a reduced space of solutions as well as its connection with the Hamiltonian framework is extensively discussed in Belot [2007].

${ }^{19}$ In this respect general relativity would seem to be identical to nonrelativistic theory. Belot's argument (which was designed for application to GR) will be explicitly re-examined for the case of relativistic theory in a companion paper.

${ }^{20}$ Rather we should say that first class constraints indicate the presence of gauge symmetries but need not necessarily be identified as the relevant generators. This point is in full agreement with Barbour and Foster [2009]
} 
temporal machinery provided by a reduced phase space. The definition of a DiracBegman observable also becomes ambiguous within timeless theory since by application of the third condition from $\S 3.4$ observable functions must be equivalent to single points on our reduced phase space - and this would seem to trivialise them. Furthermore, the first condition (constancy along gauge orbits on the constraint manifold) can only be satisfied in the case of phase space functions which are constant along entire histories of the system and it is difficult to see how such functions - perennials in the terminology of Kuchař [1992] - could be used to represent dynamic physical quantities since they cannot change along the solutions defined by the Hamiltonian on the constraint surface. Thus we are also presented with a problem of representing observables. This section will outline and evaluate two methodologies each designed to meet our two problems for the case of nonrelativstic theory.

\subsection{The emergent time strategy}

That the Hamiltonian constraint in reparameterization invariant theories should be thought of as generating genuine change is a position that has been notably defended by Kuchař [1991] and Barbour [1994]; more recently it has been outlined explicitly in Barbour and Foster [2009]. We shall call it the Kucharr-Barbour-Foster position with regard to change. In keeping with our discussion in $\S 4.4$ it is an explicitly non-reductive strategy since it involves us treating the differences between points on the integral curves corresponding to the Hamiltonian vector field as genuine physical change. Parallel, although logically independent, to this position with regard to change is the view that observable functions need not commute with the Hamiltonian - we shall call this view the Kuchař-Barbour-Foster position with regard to observables. This explicitly nonreductive strategy characterises observables as full functions on the unreduced phase space which are allowed to break all three of the Dirac-Bergman criteria. Essential to the practical viability of this position is the possibility of quantifying the change of an observable in a gauge invariant manner and we shall here outline the methodology for doing this uniquely by using an emergent notion of time following Barbour and Foster [2009].

From above we have that a generic timeless Hamiltonian will be of the form:

$$
\begin{aligned}
H & =N h \\
h(p, q) & =0
\end{aligned}
$$

If we take a function on phase space $g(p, q)$ which we would like to interpret as corresponding to some physical quantity then, since the full phase space is a symplectic manifold, we can define the Poisson bracket of this function with the Hamiltonian function, $\{g, H\}$. This is equivalent to the Lie derivative of the function with respect to the Hamiltonian vector field, $\mathcal{L}_{X_{H}}(g)$. Since the Lie derivative is an operation on scalar functions that gives us the change of the function along a vector field $\mathcal{L}_{X_{H}}(g)$ is equivalent to a real number representing the rate of change of $g$ along the Hamiltonian vector field with respect to an arbitrary parameter $\tau$ :

$$
\frac{\delta g}{\delta \tau}=\{g, H\}
$$

Thus an infinitessimal change in the function along the vector field is equivalent to:

$$
\begin{aligned}
\delta g & =\delta \tau\{g, H\} \\
& =\delta t\{g, h\}
\end{aligned}
$$


where we have introduced the temporal increment $\delta t=N d \tau$. Crucially, we have from the invariance of the canonical action that $N d \tau$ must be invariant under reparameterizations. Since the Poisson bracket must be a real number $\delta g$ must itself also be a reparameterization invariant quantity. However, it cannot yet be taken to represent the change in a physical quantity; we have not made any restriction to the constraint surface so we have not excluded change that takes us from accessible to inaccessible states. To resolve this we introduce the weak inequality and the infinitesimal change of a dynamic variable along a physical history can be then represented as:

$$
\delta g \approx N d \tau\{g, h\}
$$

We can put this result in the context of our geometric discussion since we have that: i) the Hamiltonian can be taken to generate an equivalence class of vector fields, $X_{N h}$ upon phase space ${ }^{21}$; ii) the integral curves of each of the vector fields will correspond to the same set of solutions only with a differently scaled parameter $\tau$ marking out change along them; and iii) a reparameterization is then the map between one vector field and another (between one solution and another) by re-scalings $\tau$. Such a change is between different objects both generated by $H$ but is not strictly generated by $H$ itself. Thus it should come as no surprise that there is a viable methodology for gauge invariantly using the vector fields associated with the unreduced Hamiltonian to solve our problem of representing both change and observables in timeless theory.

Although on we now have a valid methodology for representing the change of a function along a timeless solution there does still seem to be a problem. If we were to consider astronomers in two nonidentical isolated sub-systems each using these equations to describe the dynamics of their solar system, they would end up arriving at two different measures of change since each will have to make an arbitrary choice in the form of the lapse and parameter $\tau$. However, if we make the restriction that we are dealing with closed systems of fixed energy then we are justified in fixing the form of the lapse in accordance with Jacobi's theory - i.e. such that $N=\sqrt{\frac{T}{(E-V)}}$. This Jacobi lapse allows us to define a uniquely distinguished and reparameterization invariant Newtonian temporal increment ${ }^{22}$ :

$$
\delta t=\sqrt{\frac{T}{(E-V)}} d \tau
$$

Furthermore, this Newtonian temporal increment is such that it can be defined based purely upon change in the configuration variables as:

$$
\delta t=\sqrt{\frac{\delta q_{i} . \delta q_{i}}{2(E-V)}}
$$

and we can therefore represent the change in a function along a solution without reference to the parameterization. This means that we can treat time as something which naturally emerges from the dynamics and is thus ontologically secondary to the change of configuration variables.

\footnotetext{
${ }^{21}$ We get an equivalences class rather than a unique field because the multiplier $N$ is arbitrary

${ }^{22}$ As pointed out by Barbour ([1994] §4) this privileged time measure derivable from dynamics of a closed system is equivalent to the astronomers notion of emphsesis time.
} 


\subsection{The correlation strategy}

An alternative, and perhaps more radical, methodology for representing change and observables in timeless mechanics places emphasis on the idea of correlations and may be traced back through a linage featuring famous names such as DeWitt [1967], Bergman [1961], and (arguably) Einstein [1916]. Here will will present a particular implementation of the correlation strategy which follows on from Rovelli's ([1990], [1991], [2002], [2004]) complete and partial observables methodology and is due to Dittrich ([2006], [2007]) and Thiemann ([2007]). We shall focus initially on this correlation strategy as addressing the problem of representing observables in isolation from the problem of representing change and shall designate the position outlined Rovelli-Ditterich-Thiemann observables position.

An essential element of this scheme is the move away from a representation of change in an observable as the variation of a phase space function along a history. Rather, we focus upon the configuration variables themselves (the partial observables) and assert that quantities we should be interested in endowing with physical meaning are the relations between them (the complete observables) ${ }^{23}$. Change in an observable can then be represented as the reparameterization invariant specification of the value of one configuration variable with respect to another - as correlations between partial observables. The complete observables are the families of correlation functions which individually give the value of one of the partial observables when the other (the clock variable) is equal to some real number.

A simple example will illustrate the important elements of this scheme. We can consider a system described by two configuration variables (partial observables) $q_{1}$ and $q_{2}$ which together with their conjugate momenta obey a Hamiltonian constraint of the form $H\left[q_{1}, q_{2}, p_{1}, p_{2}\right]=0$. The phase space, $\left(q_{1}, q_{2}, p_{1}, p_{2}\right) \in \Gamma$, will as usual have a symplectic structure. We can use the relevant symplectic form to define the action of the Hamiltonian vector field on an arbitrary function, $X_{H}(f)=\omega\left(X_{f}, X_{H}\right)=\{f, H\}$. The flow, $\alpha_{H}^{\tau}$, generated by this vector field can then be defined for every $x \in \Gamma$ and we can see this flow as acting on a phase space function, $\alpha_{H}^{\tau}(f)(x)$, such that it takes us along the solutions ${ }^{24}$. For our system therefore we calculate $\alpha_{H}^{\tau}\left(q_{1}\right)\left(q_{1}, q_{2}, p_{1}, p_{2}\right)$ and $\alpha_{H}^{\tau}\left(q_{2}\right)\left(q_{1}, q_{2}, p_{1}, p_{2}\right)$. We then designate one of our variables as a clock variable and seek to invert an expression of the form $T_{x}(\tau)=\alpha_{H}^{\tau}\left(q_{1}\right)(x)$ such that solving $T_{x}(\tau)=s$ for $s \in \mathbb{R}$ will give us an expression for $\tau$ in terms of $s$ and $q_{1}$. In general this inversion will only be possible for a specific interval - thus the clock variables are typically going to be at best locally well defined and so are unlikely to be continuous on phase space and this means that the scheme will be difficult to implement in practice. We can then insert the inverted expression into the second flow equation $\alpha_{H}^{\tau}\left(q_{2}\right)(x)$ by substituting for $\tau$, and produce an expression for the which (within the interval specified) gives us the value of $q_{2}$ when $q_{1}$ takes the value $s$. This complete observable represents a family of functions (one for each $s$ ) each of which expresses the correlation between our two partial observables without reference to parameterization.

Importantly, not only are complete observables families of reparameterization invariant objects but the functions on phase space that each correlation defines will commute with the Hamiltonian constraint. This means that they explicitly fulfil the second condition for a Dirac-Bergman observable and demonstrates the fundamental difference

\footnotetext{
${ }^{23}$ There is some debate as to how we should interpret the partial observables see Thiemann ([2007] p. 78), Rickles ([2008] pp.154-68) and Rovelli ([2007])

${ }^{24}$ See Dittirich [2007] eq. 2.5, 2.6 and 2.7 for explicit formulas
} 
between the Rovelli-Ditterich-Thiemann and Kuchař-Barbour-Foster positions with regard to observables. We can consider the extent to which the complete observables satisfy the other two criteria. The first condition was that Dirac-Bergman observables are functions which are constant along the orbits generated by the constraint on the constraint surface. By definition the flows generated by the Hamiltonian constraint in the phase space and the integral curves of the relevant null vector field will coincide on the constraint surface. Since each of the correlations that make up a complete observable are defined for a specific value of the flow parameter these functions do not vary along this flow and are therefore constant along gauge orbits. But it must be noted that the sense in which these functions satisfy this condition is somewhat different from the generic case in two senses. First, in a typical gauge theory an observable would be constant along gauge orbits but it would also vary between them - it is this variation off the orbits that we would normally consider physical change. Second, the sense in which they are constant on gauge orbits is almost trivial - they are each defined for a particular value of the flow parameter so in effect they establish the correlation at a particular point along an orbit. Clearly such a specification is valid all the way along the orbit only in the same strange sense that the statement 'Caesar crossed the Rubicon in $49 \mathrm{BC}^{\prime}$ is also valid for Sydney in $2010 \mathrm{AD}$.

Application of the third Dirac-Bergman condition is more acutely problematic. Since the functions that define them do not vary between gauge orbits complete observables are each equivalent to single points rather than functions on reduced phase space. This means that if we take the symplectic reduction ontologically seriously (i.e. treat the reduced phase space as primitive) we will only be left with a single correlation specified by each complete observable rather than an entire family of correlation functions since it is only through the pull back to the constraint manifold that these correlations are defined. It would seem, therefore, that there is some motivation for setting aside the Dirac-Bergman notion of an observable altogether - complete observables are defined in such a way that it is no longer fully appropriate and the Rovelli-Ditterich-Thiemann position should be seen as a distinct alternative rather than a innovative application of the orthodoxy.

We can now finally turn the the problem of change. Here we appear to have a problem since Rovelli and Dittrich hold both that evolution generated by the Hamiltonian is gauge ${ }^{25}$ and that the entire orbit it generates is what should be considered physically real. ${ }^{26}$ If we dispense with the first proposition (which clearly must contradict the non-reductive stance taken by these authors) and focus on the second, then a coherent but highly radical position to emerges. In particular, if we consider the implications of the change in the notion of the physical state that seems to have been made, then it appears that the Rovelli-Dittrich-Thiemann position with regard to change in nonrelativistic reparameterization invariant mechanics amounts to a denial of the need for any fundamental concept of time at all.

Rovelli [2002] distinguishes the 'physical phase space' as the 'space of orbits generated by the constraints on the constraint surface' (p3) and Dittrich [2007] similarly defines the physical state as an 'equivalence class of phase space points' which 'can be identified with an $n$-dimensional gauge orbit' ( $\mathrm{p}$ 1894). For a theory where the Hamiltonian is itself a constraint this constitutes a redefinition of the structure of our dynamics such that the basic ontological entity is an entire history rather than an instantaneous

\footnotetext{
${ }^{25}$ See Rovelli ([2004] p. 127) and Dittrich ([2007] p.1892). Thiemann's ([2007] p.75) position with regard to this point is more nuanced and is specifically targeted to the case of general relativity.

${ }^{26}$ See Rickles ([2008] pp.182-186) and Dittrich ([2007] p.1894)
} 
configuration. In typical gauge theories points on the constraint surface connected by a gauge orbits are classified as the same state because the difference between them is taken to be unphysical - we can then proceed to a symplectically reduced phase space within which we can characterise the change between two instantaneous states without problem. This interpretation of change drawn from the complete observables scheme on the other hand leads us to classify two such points as the same state because the word state is redefined such that in includes all points on the orbit. This is not to classify time or evolution as gauge since that would indicate that the trivial reduced phase space of single initial data points was the arena of true physical significance. Rather it is to adopt a position such that any notions of evolution and time in a conventional sense are redundant within reparameterization invariant theory. Adoption of a correlation strategy has then the capacity for radical philosophical implications for the nature of time in physical theory - the next section will examine these in more detail as well as considering the emergent time strategy in a more philosophical context.

\section{$6 \quad$ Interpretational Implications}

The more strictly analytical object of this paper has been to demonstrate that unlike standard gauge theories, timeless non-relativistic theories are such that the constraints cannot be considered as gauge generators without trivialization and that a reduced phase space with a symplectic geometry cannot be constituted autonomously. In this context we have examined two strategies for representing observables and change in the unreduced phase space and considered some of the implications of each scheme. What now concerns us is to evaluate the interpretational consequences we should attach to our conclusions. In particular, it is interesting to consider how we should place the existence of; 1) gauge theories with suitably non-reducable phase spaces and 2) our two strategies for representing change without an explicit notion of time; in the context of the debates over both relationalism/subsantivalism with respect to time and reductionism/non-reductionism with respect to the interpretation of gauge theories.

\subsection{The relationalist vs substantivalist dispute with regard to time}

The long standing relationalist/substantivalist dispute with regard to space and motion in non-relativistic mechanics contains many important lessons for the parallel dispute with regard to time. In particular, modern treatments in terms of analytical mechanics allow us to precisely characterize a number of refinements to the traditional binary distinction - we will very briefly introduce the ideas key for our purposes, a more exhaustive analysis can be found in Rickles [2008].

Let us define a substantivalist as someone who is committed to the existence of space (or space-time) as an entity in its own right, over and above the relations that hold between material bodies. The position of a straightforward substantivalism is then cashed out in terms of a commitment to the existence as distinct individuals of space (space-time) models which differ only by the application of an element of the Euclidean (Galenian) group of global symmetry transformations. In terms of the Hamiltonian formulation of mechanics this is to insist that points in phase space which are related by symmetry transformations can represent distinct instantaneous states despite being empirically indistinguishable - standard substantivalism thus involves taking a Haecceitist line with regard to phase space seen as possibility space structures. 
A sophisticated substantivalist is someone who maintains the commitment to ontological fundamentality of space (or space-time) but does not insist that models related by symmetry transformations are distinct individuals - rather the individual is multiply realized within symmetry related models. We can therefore understand sophisticated substantivalism in terms of an anti-Haecceitist position with regard to phase space with the equivalence class of points connected by the relevant symmetry transformation constituting realizations of the same possibility.

The relationalist on the other hand wants to deny that space (space-time) is a fundamental entity and is therefore committed to disavowing any ontological distinction between models which differ only with regard to space (space-time) symmetries. This leaves open two options with regard to the relevant possibility spaces of the Hamiltonian re-formulation of Newtonian mechanics; either endorse anti-haecceitism and stick with the original phase space - this is what Rickles calls unsophisticated relationalism or move to a quotient space where points related by elements of the relevant symmetry group reduced to single points. This second option is what we will call reductive relationalism and, like reductionism with respect to gauge theory, is notably advocated by Belot ([1996],[1999], [2000]).

With these distinctions in hand, and the existence of a connection between reductionism and relationalism already apparent, we can turn our attention to the ontological status of time within our timeless theories of non-relativistic mechanics. We can define a temporal substantivalist as someone who asserts the existence of time as a basic entity in its own right over and above the relations that exist between the instantaneous states of material systems (be they relationally defined or not). Such a position is a natural reformulation of the Newtonian concept of absolute time in particular it seems to clearly implement that notion of time defined in the influential Scholium section of his Principia ${ }^{27}$. Now it could be argued that, at least as non-relativistic mechanics is concerned, substantivalist time is inherently connected to the use of an external temporal dimension and on this basis a substantivalist would will have a very hard time dealing with Jacobi's theory. However, what is essential to temporal substantivalism under our reading of it at least - is that time can be asserted as a basic entity parameterizing change that is not parasitic on the motion of the bodies that are doing the changing. Thus, Jacobi's theory does not in principle exclude temporal substantivalism since change is parameterized (albeit non-unqiuely) in terms of $\tau$. Moreover, unlike its Newtonian counterpart (as well as parameterized particle mechanics) Jacobi's theory offers a level playing field for matching the temporal substantivalist against their relationalist foe since it is a mechanical framework free from the fundamental presumption of preferred parametrization that would inherently favour a substantivalist reading.

A straightforward (i.e. haecceitist) substantivalist reading of Jacobi's theory could then proceed as follows. Just as the reality of space indicates that there is a real but non-qualitative difference between two points in velocity-configuration/phase space that differ only with regard to the application on an element of the Galilei group of global space-time symmetries, the reality of time indicates that there is a real but non-qualitative difference between two ways of parameterizing the dynamical change between points. That these conceptions of time are connected by an element of the local symmetry group of time reparameterizations does not mean that they fail to

\footnotetext{
${ }^{27}$ 'Absolute, true, mathematical time, of itself, and from its own nature, flows equably without relation to anything external, and by another name is called duration: relative, common time, is something sensible and external (whether accurate or unequal) measure of duration by which the means of motion, which is commonly used instead of true time' Newton [1687]
} 
be distinct individuals because, even though such a symmetry means their can be no empirical difference between worlds which contain the two times, our acceptance of primitive identity allows us to say that there is an ontological difference. Thus the straightforward substantivalist type position with respect to time in Jacobi's theory leads us to a commitment to haecceitism with regard to the parametrization of solutions rather than points. Correspondingly, Jacobi's theory, at least as formulated in section 5.3 , leaves open the conceptual space for a sophisticated (i.e. anti-haecceitist) form of temporal substantivalism whereby time is still asserted as a basic ontological entity but is multiply realized in terms of the different parameterizations of a solution - a single fundamental notion of time can be understood as being represented by the equivalence class of parameterizations.

A temporal relationalist can be defined as someone who treats time as a nonfundamental or derived entity. Such an anti-Newtonian position is typically seen to have originated with the work of Descartes, Leibniz and perhaps also Hugens ${ }^{28}$ but is contained in the most direct form within the ideas of Mach ${ }^{29}$. With regard to Jacobi's theory temporal relationalism should be understood as an insistence that that the parametrization of a solution is unphysical since it is only the relation between two instantaneous states which should matter not how the this relation is 'abstracted' in terms of parametrization ${ }^{30}$. Thus, just as the spatial relationalist was committed to two points in velocity-configuration/phase space which are connected by spatial symmetries representing the same thing, the temporal relationalist is committed to two parameterizations of a solution within the relevant space representing the same thing. This would seem, prima facie, to leave open the option for either a unsophisticated variant of temporal relationalism whereby we merely utilize anti-haecceity to 'mop-up' the excess possibilities entailed by the multiplicity of parameterizations or a reductive variant whereby we we quotient out the relevant symmetry group to leave a space with the requisite reduced set of possiblities.

So far the debate seems to closely resemble that for space/space-time. However there are two new and interesting complications that we must consider. The first stems from the fact that the reparameterization symmetry of Jacobi's theory is, unlike the global symmetries that feature in the space/spacetime debate, manifestly local. The locality of the symmetry means that a straightforward substantivalist who sticks with haecceity with regard to points in an unreduced possibility space could be left open to pernicious indeterminism in their ontology of the type discussed in section 5.4. Such a development has been key to the perceived derailment of straightforward substantivalism for the case of general relativity which features local space-time symmetries ${ }^{31}$ and may be expected for this case also. Of course, our straightforward temporal substantivalist has only thus far be understood to be committed to the haecceity of parameterized solutions in the relevant possibility space. However, like the corresponding position for points local symmetries have the potential to generate a ontological undedetermination issue for people who assign primitive identity to parameterized curves. Consider the case of a system represented by possibility space which features two solutions which are connected by an element of the reparameterization group. These two solutions

\footnotetext{
${ }^{28}$ Barbour (unpublished)

${ }^{29}$ 'It is utterly beyond our power to measure the changes of things by time. Quite on the contrary, time is an abstraction, at which we arise by means of the change of things.' Mach [1883]

${ }^{30} \mathrm{Such}$ a definition is in full accordance with the notion of a 'Leibnizian relationalist' with respect to time found in Pooley and Brown [2001]

${ }^{31}$ This is in fact the essence of the hole argument see Rickles ([2008] section 4-5) for a more extensive discussion
} 
are of course physically indistinguishable and yet there is no restriction such that if their paths through the possibility space initially coincide then they must continue to coincide. Thus, if we are invested in the primitive identity of each and every solution we end up with the potential for running into pernicious indeterminism of the same variantly that besets attempts to read the points in the phase spaces of gauge theories haccesitically.

Now, the case of Jacobi's theory is particularly interesting because although pernicious indeterminism is present within the velocity-configuration space of Jacobi's theory - since the velocities are dependent on parametrization - it is not found within the phase space since reparameterizations are symmetries on the canonical momenta. This means that provided they confine themselves to the constraint manifold a temporal substantivalist can stick to a completely haecceitist reading of both curves and points in phase space with each point representing a distinct instantaneous state and each solution representing a distinct dynamical history. Thus even though Jacobi's theory cam be classified as a gauge theory, in that it features first class constraints, it has a phase space that can unproblematically accommodate a non-reductive interpretation without any recourse to anti-haecceitism. In this respect it constitutes a notable counter example to accounts of the interpretation of gauge theories (such as that presented by Belot and Earman [1999], [2001]) which are presumed by their authors to hold generically.

The second point that marks the substantivalism vs relativism dispute with regard to time in Jacobi's theory distinct from both the case of global symmetries in Newtonian mechanics and local symmetries in generic gauge theories is that the a reductionist position is no longer available. As discussed extensively above, the structure of Jacobi's theory is such that the application of symplectic reduction will lead to a reduced phase space which has a trivial dynamical structure such that it can only be made sense of by reference back to the unreduced space. This renders a reductionist reading of the theory inadequate since to get off the ground it would require the utilization of exactly the otiose structure (gauge related points on the constraint manifold) the elimination of which was its supposed benefit. Moreover, the reductionist desire to construct a reduced phase space which can be interpreted along literal (i.e. haecceitist) lines manifestly fails since on its own the relevant reduced space can only be read as representing isolated instantaneous states corresponding to dynamically trivial universes. Thus, with regard to time in Jacobi's theory at least any viable form of relationalism is going to have to be non-reductive - does this then mean that it must be anti-haecceitist? In order to answer this question let us then consider the relationalist credentials our two non-reductive strategies for representing change and observables. In particular, it is interesting to consider how we should interpret their presentation of dynamics constraint manifold in terms of the ideas of possibility spaces and haecceity that we have been discussing.

\subsection{An ontology of timeless change?}

As discussed above the emergent time strategy explicitly makes use of the Hamiltonian constraint as the generator of evolution. A point on the constraint manifold is taken to represent an instantaneous state and the dynamical change between this state and the next is represented in terms of the null vector corresponding to the flow generated by the Hamiltonian at that point. Similarly, an observable is represented by a function of the constraint manifold and the change in an observable is represented by the change in that function along the Hamiltonian flow. Now, it has been argued by Belot 
and Earman ([1999], [2001]) that for the case of general relativity treating the relevant Hamiltonian constraint in such a manner (in particular allowing for observables that do not commute with the Hamiltonian constraint) is the the hall mark of a Heraclitean position that asserts the fundamentality of time within the theory. Conversely, according to this view point there is an equivalence between treating the Hamiltonian constraint as gauge generating (and therefore implementing the Dirac-Bergman criteria for observability) and relationalism. Clearly, adopting such a classification scheme for Jacobi's theory would seem to suggest that we should think about the emergent time strategy in terms of temporal substantivalism. Pooley [2001] argues that we should adjust this classification scheme such that how we treat the relevant constraints of general relativity is now thought of as a guild as to deciding between 'straightforward substantivalism on the one hand and the disjunctive set of sophisticated substantivalism and antisubstantialism relationalism on the other' (p. 15). Thus, under Pooley's scheme the emergent time strategy for understanding change in Jaocbi's theory would be classed as a straightforward substantivalist one with respect to time. However, as has been argued for the case of general relativity (see Rickles [2007], p. 170) the assertion of such definite connections between the treatment of the observables/Hamiltonian constraint and substivalist/relationalist distinctions is not in fact justified. There is more potential for metaphysical undedetermination within the formalism that would appear at first sight. The crucial factor informing Pooley's division is the division between treating objects relevant by the relevant symmetry as distinct individuals or not. For the case Jacobi's theory (and actually also in GR itself - see future work for detailed argument) this turns on how we understand solutions related by the relevant gauge symmetry and not points connected by the action of the Hamiltonian constraint. In Jacobi's theory one can happily avoid straightforward substantivalism whist still denying that the Hamiltonian constraint generates gauge so long as you describing the change of observables (which themselves may fail to respect the Dirac-Bergman creieria) without reference to parametrization - it is change in parametrization that we want call unphysical not the change that is parameterized! The emergent time strategy is temporally relational since it has removed temporal structure altogether and allows us to describe change, both of observables and states, without reference to parameterizations. Moreover, it has no need for the anti-haecceitism of unsophisticated relationalism since it can make use of a one-to-one representational relationship between points and instantaneous states on the one hand and solutions uniquely parameterized via the Newtonian temporal increment and dynamical histories on the other. As such it is in fact a irresistibly temporally relational mechanical framework since their is simply no temporal entity available for the substantivalist to reify - in effect a reduction of the possibilities entail by the multiplicity of parameterizations has been performed. However, this reduction is done by use of the Newtonian temporal increment rather than by a direct geometric reduction of the relevant symmetry.

The correlation strategy is distinguished by providing a reparameterization invariant description of the change of observables which satisfies the second Dirac-Begrman criterion of commuting with the constraints but does not make explicit recourse to the reduced space a la reductionism. However, as discussed at the end of the last section it leads us to a notion of change which constitutes a radical departure from that used in conventional physical theory. The notion of an instantaneous state is dispensed with and the observables are smeared non-locally along an entire solution as constituted by the gauge orbit of the Hamiltonian constraint on the constraint surface in phase space. Thus, like in an anti-haecceitist reading of a possibility space structure 
in gauge theory there is a representational correspondences between an equivalence class of gauge related points and the fundamental individual entity. However, unlike under an anti-haecceitist viewpoint the correlation scheme does not treat these points as multiple realizations of the same individual but rather as a collected realization. The fundamentally original manoeuver is to redefine the idea of a state such that it is closer to the idea of a history than its original meaning. How should we see the correlation scheme in the context of our various forms of relationalism and substantivalism? Clearly it cannot be interpreted in terms of temporally substantivalist ontology since time or even change in the traditional sense do not feature in the relevant formalism. It is also incompatible with reductive relationalism since it utilizes the un-reduced phase space space, nor can it be interpreted as in unsophisticated relationalist terms because it does not make use of anti-haecceitist methodology for connecting equivalence classes with individuals.

Rather, we must consider the possibility that the correlation strategy cannot be naturally interpreted in terms of either a relationalist or substantivalist ontology. If we take the issue of primacy between temporal structure and the relations between instantaneous states of a material system to demarcate distinction between temporal relationalism and substantivalism then the clearly a theory in which there are no instantaneous states or temporal structure will transcend our system of classification. If we define temporal relationalism to simply mean 'not temporally substantivalist' then we can happily think of the correlation scheme as relationalist - but if we are to more constructively think about temporal relationalism in terms of its Machian philosophical underpinnings with the concept of time parasitic on relational change then the correlation scheme is defiantly not relationalist with regard to time since even a derived, relational notion of time cannot be found within the formalism. What kind of ontology should we give to the correlation scheme then if not a temporal relationalist one? The most obvious option would be to take a starkly Paramedian one - time is purely an illusion and not even a derived or emergent phenomena. There is no change or evolution, merely correlations and timeless states corresponding to histories which cannot be temporally decomposed into instants. In the context of non-relativistic mechanics being adopting such a radical notion of timelessness would seem undesirable given other options are viable and this; together with the issue of practical applicability; would seem to push us away from adopting the correlation strategy. For addressing the problem of representing change and observables in nonrelativistic timeless mechanics the emergent time strategy clearly provides us with a better option since its interpretation consequences are far more palatable. The case of general relativity, however, is another matter, and the that arena radical timelessness may become a necessity.

\section{Preview of the case of general relativity}

In the preceding analysis of time, symplectic reduction and nonrelativistic timeless mechanics we established a case within which the conventional interpretation of the Hamiltonian constraint as a gauge generator can be seen to fail. We then moved on to examine the consequences of this failure both by investigating two methodologies for representing observables and change without reduction and examining the relevant interpretational consequences . Since similar constraints also appear within canonical general relativity one would be tempted to apply many of our conclusions to the relativistic case without much further analysis. However, this would be inappropriate since a number of complications within this more powerful theory must be considered 
in detail before our argument can run though. In particular, the simple correspondence between the Dirac-Bergman theory of constraints and straightforward symplectic reduction procedure discussed above breaks down for a theory, such as GR, which features both primary and secondary constraints. Moreover, although general relativity does feature an infinite set of Hamiltonian constraints, which together play a role similar to the single Hamiltonian constraint we have encountered in nonrelativistic timeless theory, these Hamiltonian constraints are non-trivially related both to each other and a second set of constraints (called the momentum constraints) which arise in the theory. A full exploration of the algebra (which in fact fails to be a Lie algebra) constituted by the various constraints is a necessary precondition of a meaningful analysis of the symmetries of general relativity and goes beyond the level of our current discussion ${ }^{32}$.

We can at least, however, reconstruct certain key elements of our argument. The phase space of GR, $\Gamma$, is a Poisson manifold and it is therefore equipped with a canonical (four) form ${ }^{33} \tilde{\theta}$. The constraints of the theory (which will not be discussed in detail here) define a constraint surface, $\Sigma$, and the restriction of our original form to this surface will induce a second form $\theta=\left.\tilde{\theta}\right|_{\Sigma}$. The exterior derivative of this gives us a (five) form $\omega=d \theta$ and since $\omega$ has null directions it is presymplectic and therefore defines a presymplectic geometry $(\Sigma, \omega)$. As for the nonrelativistic case points in the phase space not on this surface correspond to inaccessible/unphysical instantaneous states of the world. Even more significantly, if we define the orbits of $\omega$ to be four dimensional surfaces $\bar{\gamma}$ in $\Sigma$ such that the quadritangent $X$ to the orbit is in the kernel of $\omega$ (i.e. $\omega(X)=0$ ) then we can identify the $\bar{\gamma}$ with solutions of the Einstein field equations. This indicates that as for our nonrelativistic timeless theories general relativity has the unusual geometry such that the degeneracy and dynamics are entangled - what we would normally consider gauge orbits are in fact the physical solutions themselves. Thus, the application of symplectic reduction (and therefore the conventional application of Dirac's theory of constraints) is also going to be at the very best problematic and at worst trivialising in the case of general relativity. It would seem reasonable, then, to assume that whatever the precise interpretation of the Hamiltonian constraints of general relativity we are unlikely to be able to construct its dynamics in terms of a reduced symplectic manifold. Our two timeless strategies for addressing the problem of representing change and observables within a presymplectic geometry would thus appear particularly relevant to the case of general relativity. Their evaluation in the context of the full theory of relativity together with the appropriate interpretational analysis will be the subject of future work.

\section{A Elements from differential geometry}

This appendix contains only a very brief review of the key definitions of concepts from differential geometry used in the paper. See Baez and Muniain [1994] or Butterfield [2007] for a detailed introduction.

A Lie group, $G$, is a group ${ }^{34}$ that is also a differentiable manifold ${ }^{35}$ with the property that the product and inverse operations are smooth (i.e. have continuous

\footnotetext{
${ }^{32}$ See Isham [1992], Kuchař [1986] and especially Thiemann ([2008], §1.4)

${ }^{33}$ See Rovelli ([2004] $\$ 4.3$ ) for an explict construction of GR in these terms.

${ }^{34} \mathrm{~A}$ set of elements, $g$, with an identity element, $e$, within which an operation of combining elements to get another element also in the set (i.e. $g_{3}=g_{1} \circ g_{2}$ and $g=g \circ e$ ) is defined such that it is associative and within which each element has an inverse (i.e. $g \circ g^{-1}=e$ )

${ }^{35} \mathrm{~A}$ space that is locally similar enough to Euclidean space for us to be able to do calculus on it.
} 
derivatives). The action of a Lie group on a manifold, $\Phi(g, x)$ or $g \cdot x$ where $x \in M$, is a smooth map $\Phi: G \times M \rightarrow M$ that implements the identity and associative aspects of the group. The orbit of the action through a point on a manifold is a set of points $[x]:=\{g \cdot x: g \in G\}$. Under certain conditions the action of $G$ on $M$ is to define a foliation of $M$ with the orbits as the leaves of the foliation ${ }^{36}$. We can form a set $N=M / G$ know as the quotient ${ }^{37}$ of $M$ by the group $G$ by considering the set of orbits of the action of $G$ for every point in $M$ i.e. $N:=\{[x]: \forall x \in M\}$.

The simplest Lie group is the additive real group $\mathbb{R}$. It defines an $\mathbb{R}$-action $\Phi$ : $\mathbb{R} \times M \rightarrow M$ and we associate it with a one parameter group of diffeomorphisms from $M$ to $M$ called a flow $\left\{\alpha^{t}\right\}$ through the relation $\alpha^{t}(x)=\Phi(t, x)$ for $x \in M$. If the flow is well defined for all $t \in \mathbb{R}$ it is global, otherwise it is local. Every $\mathbb{R}$-action on $M$ induces a unique assignment of a tangent vector, $T_{x} M$, to every point in $M$ and thus allows us to define a unique tangent vector field, $X: x \in M \longmapsto X(x) \in T_{x} M$. Conversely we can think of a given vector field as generating both an $\mathbb{R}$-action and a flow. Given a vector field on a manifold we can define a family of integral curves as smooth maps, $\gamma_{x}(t): I \rightarrow M$, from a real open interval $I \subset \mathbb{R}$ to $U \subset M$, by considering the local flows (global if $I=\mathbb{R}$ and $U=M$ ) generated through every point in the manifold (i.e. $\gamma_{x}(t): t \longmapsto \Phi_{t}(x) \forall x \in M$ ). These curves are such that each point in $M$ lies on exactly one such curve and the parameterization of each curve up to a choice of origin is fixed.

Given a vector field, $X$, on a manifold, $M$, we can define the Lie derivative, $\mathcal{L}_{X}: f \longmapsto \mathcal{L}_{X} f: M \rightarrow \mathbb{R}$, as an operation on scalar functions, $f$, that gives us the rate of change of $f$ along $X^{38}$. Given another vector field, $Y$, also on $M$ we can then define the commutator between the two relevant Lie derivatives, $\mathcal{L}_{X} \mathcal{L}_{Y}-\mathcal{L}_{Y} \mathcal{L}_{X}$, as the Lie derivative of one vector field with respect to another, $\mathcal{L}_{X} Y$. It is equal to a third vector field which we call the Lie bracket, $[X, Y]=\mathcal{L}_{X} Y$. The Lie bracket constitutes a binary operation over a vector space and therefore defines an algebra. This algebra is one instance of a special type of algebra called a Lie algebra.

\section{B Acknowledgments}

Thanks to Julian Barbour, Pete Evans, Dean Rickles, Carlo Rovelli, Hans Westman and an anonymous referee for many helpful comments on the previous draft forms of this paper.

\section{References}

[1] Abraham, R. and Marsden J. E. [1978]: Foundations of Mechanics. Benjamin/Cummings

[2] Anderson, E [2010]: The problem of time in quantum gravity, $\langle$ http://arxiv.org/abs/1009.2157〉

[3] Arnold, V.I., Kozlov, V.V, Neishtadt, A.I. [1988]: Mathematical aspects of celestial mechanics, tran. Iacob, A. Springer-Verlag

\footnotetext{
${ }^{36}$ See Souriau (1970) p. 38 and p.49

${ }^{37}$ The quotients we will deal with in this paper will be manifolds, see $\S 3.4$ for details. The general conditions for a quotient to be a manifold can be found in Souriau (1997) pp.13-14.

${ }^{38}$ Explictly, $\left(\mathcal{L}_{X} f\right)(x):=\left.\frac{d}{d t}\right|_{t=0} f\left(X^{t}(x)\right) \equiv X(x) f \quad \forall x \in M$, where $t$ is the parameter of the flow generated by $X$.
} 
[4] Arnowitt, A., Deser, S. and Misner, C. [1962) The Dynamics of General Relativity, L Witten [ed.) Gravitation: An Introduction to Current Research. John Wilet \& Sons

[5] Barbour, J. [1994]: The timelessness of quantum gravity: I evidence from the classical theory, Classical and Quantum Gravity 112853

[6] Barbour, J. B. and O Murchadha, N. [1999]: Classical and quantum gravity on conformal superspace, http://arxiv.org/abs/gr-qc/9911071.

[7] Barbour, J. B., Foster, B. Z. and O Murchadha, N. [2000]: Relativity without relativity, $\langle$ http://arxiv.org/abs/gr-qc/0012089〉

[8] Barbour, J. B. [2003]: Dynamics of Pure Shape, Relativity, and the Problem of Time, $\langle$ http://arxiv.org/abs/gr-qc/0309089〉

[9] Barbour J [unpublished] MachÕs Principle, General Relativity and Gauge Theory

[10] Barbour, J. B, and Foster, B [2009]: Constraints and gauge transformations: Dirac's theorem is not always valid. $\langle\operatorname{arXIv} ; 0808.23334 \mathrm{v} 1\rangle$

[11] Baez, J and Muniain, J. P. [1994]: Gauge Fields, Knots, and Gravity. World Scientific

[12] Belot, G. [1996] Whatever is Never and Nowhere is Not: Space, Time, and Ontology in Classical and Quantum Gravity. University of Pittsburgh Doctoral Dissertation

[13] Belot, G [1999]: Rehabilitating Relationalism. International Studies in the Philosophy of Science 13 35-52.

[14] Belot, G [2000]: Geometry and Motion. British Journal for the Philosophy of Science 51: 561-595 [50th Anniversary Issue]: Reprinted in P. Clark and K. Hawley (eds.), Philosophy of Science Today. Oxford University Press [2003), pp. 201-235.

[15] Belot, G [2003]: Symmetry and Gauge Freedom. Studies in History and Philosophy of Modern Physics 34 189-225

[16] Belot, G [2007]: The representation of time and change in mechanics. In Butterfield, J. and Earman, J. (eds.), Handbook of Philosophy of Physics. Elsevier

[17] Belot, G. Earman, J. [1999]: From Metaphysics to Physics. In J. Butterfield, and C. Pagonis (eds.), From Physics to Philosophy. Cambridge University Press, pp. 166-186.

[18] Belot, G and Earman, J [2001]: Presocratic quantum gravity. In Callender C and Huggett, $\mathrm{N}$ [eds), Physics meets philosophy at the Planck scale. Cambridge University Press

[19] Bergman, P [1961]: Observables in General Relativity. Rev. Mod. Phys. 334510

[20] Butterfield, J. [2007]: On symplectic reduction in classical mechanics. In Butterfield, J. and Earman, J. (eds.), Handbook of Philosophy of Physics. Elsevier

[21] De Witt [1967]: Quantum theory of gravity. I. The canonical theory, Physical Review, 160, $1113-1148$

[22] Dirac, P. A. M. [1958a]: Generalized Hamiltonian dynamics, Proceedings of the Royal Society of London. Series A, Mathematical and Physical Sciences, 246, 326-332 
[23] Dirac, P. A. M. [1958b]: The theory of gravitation in Hamiltonian form, Proceedings of the Royal Society of London. Series A, Mathematical and Physical Sciences 246, 333-343

[24] Dirac, P. A. M. [1964]: Lectures on quantum mechanics. Dover

[25] Dittrich, B. [2006]: Partial and complete observables for canonical general relativity, Classical and Quantantum Gravity 236155

[26] Dittrich, B. [2007]: Partial and complete observables for Hamiltonian constrained systems, General Relativity and Gravitation 391891

[27] Earman, J. [2001]: Thoroughly modern Mctaggart. Philosopher's Imprint 2 No. 3

[28] Einstein, A. [1916]: Die Grundlage der allgemeinen Relativitätstheorie, Annalen der Physik 49: 769-822. English translation from W. Perrett and G. B. Jeffrey (eds.), The Principle of Relativity. New York: Dover, 1952

[29] Gotay, M.J, Nester, J.M and Hinds, G [1978]: Presymplectic manifolds and the DiracBergman theory of constraints, Journal Mathamatical. Physics 19, 11

[30] Henneaux, M. and Teitelboim, C. [1992]: Quantization of gauge systems. Princeton University Press

[31] Isham, C. [1992]: Canonical Quantum Gravity and the Problem of Time 〈gr-qc/9210011〉

[32] Johns, O. D. [2005]: Analytical mechanics for relativity and quantum mechanics. Oxford University Press.

[33] Kuchař, K. [1986]: Canonical Geometrodynamics and General Covariance, Foundations of Physics, Vol 16 No. 3

[34] Kuchar, K. [1988]: The Problem of Time in Canonical Quantization of Relativistic Systems, Ashtekar, A. and Stachel, J (eds.), in Conceptual Problems of Quantum Gravity, Boston University Press

[35] Kuchař, K. [1992]: Time and interpretations of quantum gravity, Proceedings of the fourth Canadian conference on general relativity and relativistic astrophysics. World Scientific Singapore

[36] Lanczos, C. [1966]: The variational principles of mechanics. University of Toronto Press

[37] Newton I, [1687]: Philosophiaie Naturalis Principia Mathematica, in The Leibniz-Clarke Correspondence, ed. H.G Alexander, [1956], Bell and Bain Ltd

[38] Newman, E. and Bergman, P. G. [1957]: Observables in singular theories by systematic approximation. Review Modern Physics 29443

[39] Mach, E. [1883]: Die Mechanik in ihrer Entwicklung historisch-kritsch dargestellt, Barth, Leipzig; English translation: Mach E 1960 The Science of Mechanics, Open Court, Chicago [translation of 1912 German edition)

[40] Marsden, J and Ratiu, S. [1994]: Introduction to Mechanics and Symmetry, SpringerVerlag

[41] Maudlin, T. [2002]: Thoroughly muddled Mctaggart, Philosopher's Imprint 2 No. 4

[42] Misner, C. Thorne, K. and Wheeler, J. [1970]: Gravitation, W.H Freeman and Company

[43] Ponds, J. M. [2005]: On Dirac's incomplete analysis of gauge transformations, Studies in History and Philosophy of Modern Physics 36491 
[44] Ponds, J. M., Sailsubury, D.C. and Shepley, L. C. [1999]: Reduced phase space: quotienting procedure for gauge theories, Journal of Physics A: Mathematical and General 32419

[45] Pons, J. M. and Sailsubury, D.C. [2005]: The issue of time in generally covariant theories and the Komar-Bergmann approach to observables in general relativity, Physical Review D 71124012

[46] Readhead, M. [2003]: The interpretation of gauge symmetry. Brading, K and Castellani, E (eds.), in Symmetries in Physics: Philosophical Reflections pp. 124-139. Cambridge University Press

[47] Rickles, D. [2004]: Symmetry \& possibility: to reduce or not reduce?, 〈http://philsciarchive.pitt.edu/archive/00001846>

[48] Rickles, D. [2008]: Symmetry, structure and spacetime. Philosophy and foundations of physics Vol. 3. Elseiver

[49] Rovelli, C. [1990]: Quantum Mechanics without time: a model. Physical Review. D 428

[50] Rovelli, C. [1991]: Time in quantum gravity: An hypothesis. Physical Review D 43442

[51] Rovelli, C. [2002]: Partial Observables, Physical Review D Vol. 65124013

[52] Rovelli, C. [2004]: Quantum gravity, Cambridge University Press

[53] Rovelli, C. [2007]: Comment on "Are the spectra of geometrical operators in Loop Quantum Gravity really discrete?" by B. Dittrich and T. Thiemann, $\langle$ http://arxiv.org/abs/0708.248〉

[54] Souriau, J. M. [1997]: Structure of dynamical systems, trans. Cushman-de Vries C.H. Birkhauser

[55] Thiemann, T. [2007]: Modern canonical quantum general relativity, Cambridge Univeristy Press

[56] von Westenholz, C. [1978]: Differential forms in mathematical physics, North-Holland

[57] Weyl, H. [1939]: Classical groups: their invariants and representations, Princeton 